\title{
Changing auditory time with prismatic goggles
}

\author{
Barbara Magnani ${ }^{\mathrm{a}, *}$, Francesco Pavani ${ }^{\mathrm{b}, \mathrm{c}}$, Francesca Frassinetti ${ }^{\mathrm{a}, \mathrm{d}}$ \\ a Department of Psychology, University of Bologna, 40127 Bologna, Italy \\ ${ }^{\mathrm{b}}$ Center for Mind/Brain Sciences (CIMeC), University of Trento, 38068 Rovereto, Italy \\ ${ }^{\mathrm{c}}$ Department of Cognitive Science and Education, University of Trento, 38068 Rovereto, Italy \\ ${ }^{\mathrm{d}}$ Fondazione Salvatore Maugeri, Clinica del Lavoro e della Riabilitazione, IRCCS-Istituto Scientifico di Castel Goffredo, 46042 Mantova, Italy
}

\section{A R T I C L E I N F O}

\section{Article history:}

Received 25 July 2011

Revised 5 June 2012

Accepted 3 July 2012

Available online 3 August 2012

\section{Keywords:}

Spatial representation of time

Auditory stimuli

Prismatic adaptation

Spatial attention

\begin{abstract}
A B S T R A C T
The aim of the present study was to explore the spatial organization of auditory time and the effects of the manipulation of spatial attention on such a representation. In two experiments, we asked 28 adults to classify the duration of auditory stimuli as "short" or "long". Stimuli were tones of high or low pitch, delivered left or right of the participant. The time bisection task was performed either on right or left stimuli regardless of their pitch (Spatial experiment), or on high or low tones regardless of their location (Tonal experiment). Duration of left stimuli was underestimated relative to that of right stimuli, in the Spatial but not in the Tonal experiment, suggesting that a spatial representation of auditory time emerges selectively when spatial-encoding is enforced. Further, when we introduced spatial-attention shifts using the prismatic adaptation procedure, we found modulations of auditory time processing as a function of prismatic deviation, which correlated with the interparticipant adaptation effect. These novel findings reveal a spatial representation of auditory time, modulated by spatial attention.
\end{abstract}

(c) 2012 Elsevier B.V. All rights reserved.

\section{Introduction}

A recent theoretical framework known as ATOM (A Theory of Magnitude) theory assumes that the brain has developed an economical fashion to measure all quantities, learnt through, and useful for, interacting with the environment by action (Walsh, 2003). Such a common metric system is assumed to be inherently spatial and to have a neural substrate in the right inferior parietal cortex (Walsh, 2003; Bueti \& Walsh, 2009). Within this perspective, information concerning time would also share with other magnitudes the same spatial system of encoding. Several pieces of evidence support the latter aspect of the theory, showing that time might be spatially represented on a hypothetical mental temporal line, ascending from

\footnotetext{
* Corresponding author. Address: Department of Psychology, University of Bologna, Viale Berti Pichat, 5, 40127 Bologna, Italy. Tel.: +39 051 209 1847; fax: +39 051243086 .

E-mail address: barbara.magnani2@unibo.it (B. Magnani).
}

left to right (see Oliveri, Koch, \& Caltagirone (2009a), for a review). For instance, Vicario et al. (2008) found that the duration of visual stimulus is underestimated when it is presented in left space and overestimated when it is presented in right space, suggesting that spatial encoding can interfere with their duration processing. Specifically, there appears to be some sort of correspondence between the left-to-right spatial dimension and the short-to-long temporal dimension.

Until recently, the most part of these studies have focused on the spatial representation of time for visual stimuli (Vicario, Caltagirone, \& Oliveri, 2007; Vicario et al., 2008; Vallesi, Binns, \& Shallice, 2008; Oliveri et al., $2009 \mathrm{~b})$, raising the question of whether time processing in other sensory modalities would also show a similar spatial organization. Ishihara et al. (2008) investigated precisely this issue using a speeded response paradigm. Participants were instructed to indicate whether the timing of a probe sound was earlier or later than expected based on preceding clicks, by pressing as fast as possible a key 
lateralized to the left or to the right respectively. The results showed that left-side responses were faster for early-onset timing than late-onset timing, whereas a reverse pattern of results emerged for the right-side responses. Such a correspondence between response position and stimulus duration was only observed with horizontal, and not vertical, alignment of the response keys. These results were interpreted as evidence for a spatial representation of auditory time, horizontally aligned from left to right, which interacts with motor preparation in space. The hypothesis of a spatial representation of auditory time has now received further support by evidence in brain-damaged patients with hemi-spatial neglect (Calabria et al., 2011), showing that duration comparison between a standard tone and a test tone is worse in brain-damaged patients showing the visuo-spatial disturbance known as neglect, compared to brain-damaged patients without neglect or healthy controls.

The fact that interference between spatial encoding and time processing can emerge in a similar fashion in the visual and the auditory modality is, in some respect, surprising. In the visual modality, space is immediately available in retinotopic coordinates on the receptor surface. By contrast, in the auditory modality information is initially encoded tonotopically, and space is not immediately available on receptor surface (Barker, Plack, \& Hall, 2011; Hall \& Plack, 2009). The brain reconstructs the spatial location of an auditory stimulus based on interaural and monaural auditory cues (Blauert \& Lindemann, 1986), and the output of this computation is typically less precise than the localization of a visual stimulus. Studies on animals (Lee \& Middlebrooks, 2010; Populin \& Rajala, 2010) and humans (Pavani, Làdavas, \& Driver, 2002) have also shown that localization of sounds is most precise when the spatial encoding of the auditory stimuli is salient for the task. In cats, Lee and Middlebrooks (2010) showed that the width of spatial receptive fields (from $180^{\circ}$ to $360^{\circ}$ ) of neurons in the auditory primary cortex (A1) becomes sharper when the localization of sounds is requested by the task, as compared to when spatial factors are not salient for the animal's behavior. In humans, there is neuropsychological evidence that hemispatial neglect for auditory targets worsens when patients are asked to encode the spatial location of the stimuli, with respect to when they are asked to process the tonal aspect of the stimuli (Pavani et al., 2002; see also Deouell \& Soroker, 2000). These considerations raise the possibility that any spatial representation of auditory time would emerge more strongly whenever a spatial encoding of the auditory stimuli is enforced.

The first goal of the present study was to verify this hypothesis. To this aim, 28 young healthy participants were submitted to two time bisection experiments with auditory stimuli. Time bisection is a classical task, frequently used in the time perception literature (Wearden, 1991; Wearden \& Ferrara, 1995). It consists of the verbal classification of auditory stimuli of different duration, with respect to previously acquired pair of reference durations ("short" or "long"). In the present study, each auditory stimulus was either of high or low pitch, and it was presented to the left or to the right with respect to the participant's midsagittal plane. Most important, the time bisection task was presented as a go/no-go task. This means that, in Experiment 1 (Spatial experiment), participants were asked to classify tone durations as "short" or "long", only when the stimulus occupied a pre-determined location in space (left or right), but regardless of its pitch. This forced spatial encoding of each auditory stimulus, regardless of its pitch. In Experiment 2 (Tonal experiment), participants were asked to classify tone durations as before, but only when the stimulus was of a pre-determined pitch (high-frequency or low-frequency), regardless of its spatial location. This forced tonal encoding of each auditory stimulus, regardless of its location. The choice of the go/no-go paradigm was instrumental to selectively orient participants' attention to either the spatial or the tonal feature of the stimulus, while maintaining the stimuli completely identical between the two experiments. Participants were equally exposed to spatial and tonal variations in Experiment 1 and 2. However, by instructing participants to respond to the frequently occurring 'Go' stimuli (defined either on the basis of a spatial or tonal feature) and to inhibit responses to infrequent 'NoGo' stimuli, we ensured that our duration measures were linked selectively to the processing of either the spatial or the tonal aspect of the auditory stimulus.

Our predictions were as follows. If the spatial representation of auditory time emerges primarily (or selectively) when spatial encoding of the auditory stimuli is enforced, we expected interference of spatial location on time perception in the Spatial experiment more than in the Tonal one. More specifically, we expected duration underestimation for left sounds and duration overestimation for right sounds, in line with the hypothesis described above of a left-to-right orientation of the mental time line.

Another essential goal of the present study was to verify whether the manipulations that proved effective in changing the spatial representation of visual time would also be effective in changing representation of auditory time. One manipulation that recently proved useful in distorting the spatial representation of visual time is prismatic adaptation (PA). Prismatic lenses used in PA induce an optical deviation toward a side of space. During a pointing task to a visual target, such deviation is measured as an error in limb pointing with respect to the visual target, in the same direction as the prismatic deviation. If visual feedback about limb pointing is available, participants rapidly correct the error to compensate for the prismatic shift. Moreover, when the prismatic goggles are removed and limb pointing to the visual target is not visible, participants show systematic deviation of their pointing responses, opposite to the lenses deviation - the so-called after-effect. Several studies converge in showing that this after-effect may also result in a shift of spatial attention (Frassinetti, Angeli, Meneghello, Avanzi, \& Làdavas, 2002; Serino, Angeli, Frassinetti, \& Làdavas, 2006; Angeli, Benassi, \& Làdavas, 2004). Despite a wide literature on prismatic adaptation effects on spatial behavior, it remains unclear whether the beneficial effects of prisms arise only via the influence of adaptation on circuits controlling attention and visuomotor behaviors. However, positive effects obtained in tasks independent from any visual or motor or proprioceptive component (Jacquin-Courtois et al., 2010; 
Eramudugolla, Boyce, Irvine, \& Mattingley, 2010) and on representational neglect (Rode, Rossetti, Li, \& Boisson, 1998; Rossetti et al., 2004) are not in contrast with an interpretation of PA effects in terms of attentional shift.

Using visual stimuli, previous findings demonstrated that PA can induce spatial attentional biases toward the left or the right side of space, which in turn produce opposite measurable effects on time estimation (Frassinetti, Magnani, \& Oliveri, 2009). Specifically, duration underestimation emerged following leftward shift, and duration overestimation emerged following rightward attentional shift. If PA induces effects on time representation for auditory stimuli similar to those found on visual stimuli, underestimation of sounds duration should be expected after leftward attentional shift and overestimation of sounds duration should emerge after a rightward attentional shift.

\section{Methods}

\subsection{Participants}

Twenty-eight right-handed, Italian native-speaker students ( 5 males), aged $20-30$ years (mean age $=24.8$ years; $\mathrm{SD}=1.9$ years), were enrolled in the study. All participants were naïve as to the purpose of the study and had not history of hearing or neurological diseases. All participants gave their informed consent to participate in the study.

\subsection{Apparatus and stimuli}

Participants were tested individually in a silent and dimly lit room, with the apparatus approximately $70 \mathrm{~cm}$ in front of them. The apparatus comprised a HP laptop computer to control stimuli and collect responses, and two Olidata G-422 loudspeakers $(0.4 \mathrm{~W}, 6 \Omega)$ to deliver the auditory stimuli. With respect to the subject's midsagittal line, one loudspeaker was positioned $25 \mathrm{~cm}$ to the left and the other $25 \mathrm{~cm}$ to the right. Loudspeakers were covered by a black cloth mounted on a wooded frame, to avoid any visual cue about sound location. The auditory stimuli consisted in pure tones that could vary in fundamental frequency $(523 \mathrm{~Hz}$, corresponding to the DO5 and $262 \mathrm{~Hz}$, corresponding to the DO4 on the musical scale) and duration $(1400,1700,2000,2300,2600 \mathrm{~ms})$. Tones were presented at approximately $70 \mathrm{~dB}$ (SPL), as measured from the participant's head. The experimental program was written with E-Prime software (Psychology Software Tool Inc.), which assured millisecond accuracy for timing.

\subsection{Experimental procedure}

All participants were first trained to classify two reference tone durations (1400 and $2600 \mathrm{~ms}$ ) as short or long (practice session). Subsequently, they were presented with the full range of tone durations (1400, 1700, 2000, 2300, $2600 \mathrm{~ms}$ ) and were again asked to classify them as short or long (time bisection task). This range of tones durations, was chosen because they proved sensitive to PA effects in visual modality (Frassinetti et al., 2009) and because we wanted to focus on the cognitive controlled time perception. Classically, time perception is known to rely on cognitive systems when the estimated intervals are in the range of seconds-to-minutes (Meck, 2005; Gallistel \& Gibbon, 2000; Lewis \& Miall, 2003). Given the length of the intervals employed, participants were explicitly required not to count aloud or sub-vocally in all phases of the experimental procedure, in accordance with similar paradigms used in previous studies (Oliveri et al., 2009b).

The time bisection task was always performed according to one of two go/no-go instructions. In the Spatial experiment, participants only responded when the tone was delivered from a specific location (left or right), ignoring its pitch; in the Tonal experiment, participants only responded when the tone was of a specific pitch (high-frequency or low-frequency), ignoring its location in space. After the time bisection task, participants performed a prismatic adaptation (PA) session, in which no auditory stimulus was delivered. Finally, they repeated the time bisection task (both Spatial and Tonal experiments). In the following paragraph, all phases of the experimental session are described in details.

\subsubsection{Practice session}

The practice session served to familiarize participants with two reference durations (1400 and $2600 \mathrm{~ms}$ ). Twenty stimuli were presented in a pseudo-random order, from both loudspeakers stereophonically, giving the impression of a sound delivered straight ahead of the participant. For each duration ( 1400 or $2600 \mathrm{~ms}$ ), half of the tones were high pitch and the other half was low pitch. Participants were instructed to verbally classify the stimuli as "short" or "long", while maintaining gaze on a central fixation cross, and ignoring the tone pitch. The experimenter recorded the participant's response by pressing one of the two mouse keys (left for "short" and right for "long") and controlled that the participant complied with the fixation instruction during stimulus presentation. The practice session was repeated until the participant had reached at least $80 \%$ of accuracy. All participants reached such level of accuracy with no more than two practice sessions.

\subsubsection{Time bisection task}

The experimental procedure of the time bisection task was the same of practice session, with the following exceptions. First, the full range of tone durations was presented $(1400,1700,2000,2300,2600 \mathrm{~ms})$; second, each tone originated either from the loudspeaker on the left or from the loudspeaker on the right; third, participants responded in a go/no-go manner, as a function of stimulus location or stimulus pitch.

When responding as a function of stimulus location (Spatial experiment), participants classified the tones as "short" or "long" with respect to the reference durations, only when the stimulus occupied a pre-specified spatial location, regardless of its pitch. In one block, the participant responded only to stimuli presented on the left, ignoring stimuli presented on the right. To maximize go trials, in this block $80 \%$ of stimuli were presented on the left and $20 \%$ were presented on the right. In the other block, instructions and proportions were reversed: participants responded only to stimuli presented on the right, ignoring 
stimuli presented on the left and $80 \%$ of stimuli were presented on the right and $20 \%$ of stimuli were presented on the left. The proportion of high and low pitch tones remained equiprobabile in both these spatial blocks.

When responding as a function of stimulus pitch (Tonal experiment), participants classified the tones as "short" or "long", only when the stimulus was a pre-specified pitch, regardless of its spatial location. In one block, participants responded only to high pitch tones, ignoring low pitch ones. In this block, $80 \%$ of stimuli were high pitch and $20 \%$ of stimuli were the low pitch. In the other block, instructions and proportions were reversed: participants responded to low pitch tones, ignoring high pitch tones; $80 \%$ of stimuli were the low pitch and $20 \%$ of stimuli were high pitch. The proportion of left and right tones remained equiprobabile in both these tonal blocks.

Both the Spatial and Tonal experiment comprised 50 stimuli, resulting in 200 trials overall. The order of experiments before and after PA and of blocks within each experiment was counterbalanced across participants.

\subsubsection{Prismatic adaptation}

During PA, participants sat at a table in front of a box (height $=30 \mathrm{~cm}$, depth $=34 \mathrm{~cm}$ at the center and $18 \mathrm{~cm}$ at the periphery, width $=72 \mathrm{~cm}$ ), open on the side facing the participant as well as the opposite side facing the experimenter. The experimenter placed a visual target (a pen) at the distal edge of the top surface of the box, in one of three possible positions (randomly determined on each trial): a central position $\left(0^{\circ}\right), 21^{\circ}$ to the left of center $\left(-21^{\circ}\right)$, and $21^{\circ}$ to the right of center $\left(+21^{\circ}\right)$. Participants were asked to keep their right hand at the level of the sternum, to point to the pen using the index finger of the right hand, and then return the hand to the chest. The experimenter recorded the end position of the participant's pointing direction, by writing down the number of degrees of visual angle between the index finger and the pen position. The pointing task was performed in three experimental conditions: pre-exposure, exposure, and post-exposure. In the pre-exposure condition, participants performed two types of trials. On half of the trials, their pointing was visible to them, (30 trials) and on the other half, they could not see their pointing (30 trials). Note that these two conditions were comparable to the exposure and the post-exposure conditions described later, respectively. In the exposure condition, participants performed the task (30 trials for each spatial position: $-21^{\circ}, 0^{\circ},+21^{\circ}$ ), while wearing prismatic lenses inducing a $20^{\circ}$ shift of the visual field to the right or to the left. In this condition, they always saw the trajectory of their arm (i.e., visible pointing). In the post-exposure condition, run immediately after removal of the prisms, participants were required to make their pointing movements underneath the top surface of the box so that the index finger was never visible at any stage (i.e., invisible pointing; 30 trials). In this phase, all participants were expected to show pointing movements in the opposite direction of the visual shift induced by lenses, called after-effect. Participants were randomly divided in two groups. One group, was only exposed to prisms inducing a rightward visual shift and showed a leftward after-effect, whereas the other group was only exposed to prisms inducing leftward visual shift and showed a rightward after-effect.

\section{Data analysis and results}

\subsection{Analysis on the bisection point values}

The bisection point is the estimated temporal value (in milliseconds) for which participants would respond "long" or "short" with equal probability (Wearden \& Ferrara, 1995; Allan, 2002; Kopec \& Brody, 2010). To calculate the bisection point, we first computed the percentage of "long" responses for each interval duration $(1400,1700,2000$, $2300,2600 \mathrm{~ms}$ ), and then we fitted the percentage of "long" responses across different stimulus durations using a logistic regression. In our paradigm, the objective stimulus duration representing the medium point between the short and long reference duration was $2000 \mathrm{~ms}$. Bisection points below $2000 \mathrm{~ms}$ reflect duration overestimation (i.e., durations are perceived longer than they actually are), whereas bisection points above 2000 ms reflect duration underestimation (i.e., durations are perceived shorter than they actually are). For each participant, the bisection points were separately calculated in the tonal and spatial experiment, for each Stimulus Position (left and right) and pitch (high and low).

In order to investigate if the spatial location of the auditory stimulus interacts with auditory temporal processing, and to examine whether this is more pronounced during spatial compared to tonal encoding, we first analyzed performances in the time bisection task before PA. Subsequently the effect of PA on auditory time bisection task was assessed. Post hoc analyses were always conducted using the Least Significant Difference test (LSD test) and effect size are provided as partial eta square.

\subsubsection{Representation of auditory temporal stimuli before exposure to prismatic adaptation}

For each experiment we conducted an Analysis of Variance (ANOVA) on bisection point values before PA, using Stimulus Position (left vs. right) and Stimulus Pitch (high tone vs. low tone) as within-subjects variables.

In the Spatial experiment, a significant main effect of Stimulus Position was found $[F(1,27)=5.45 ; p=0.03$; $\left.\eta_{p}^{2}=.168\right]$. Stimuli presented on the left were underestimated compared to stimuli presented to the right ( mean $=1898 \mathrm{~ms}, \mathrm{SE}=42$ mean $=1836 \mathrm{~ms}$; $\mathrm{SE}=45$; see Fig. 1A). No other effect reached significance $(p=0.23)$.

In the Tonal experiment Stimulus Pitch $(p=0.09)$, Stimulus Position ( $p=0.80$ ) (see Fig. 1B) and their interaction $(p=0.67)$ were not significant.

\subsubsection{Effect of Prismatic Adaptation on the representation of auditory temporal stimuli}

For each experiment an ANOVA was conducted on bisection point values obtained in the experimental sessions before and after PA, using Group (LG= leftward after-effect group or RG = rightward after-effect group) as between-subjects variable and Condition (before-PA, or 

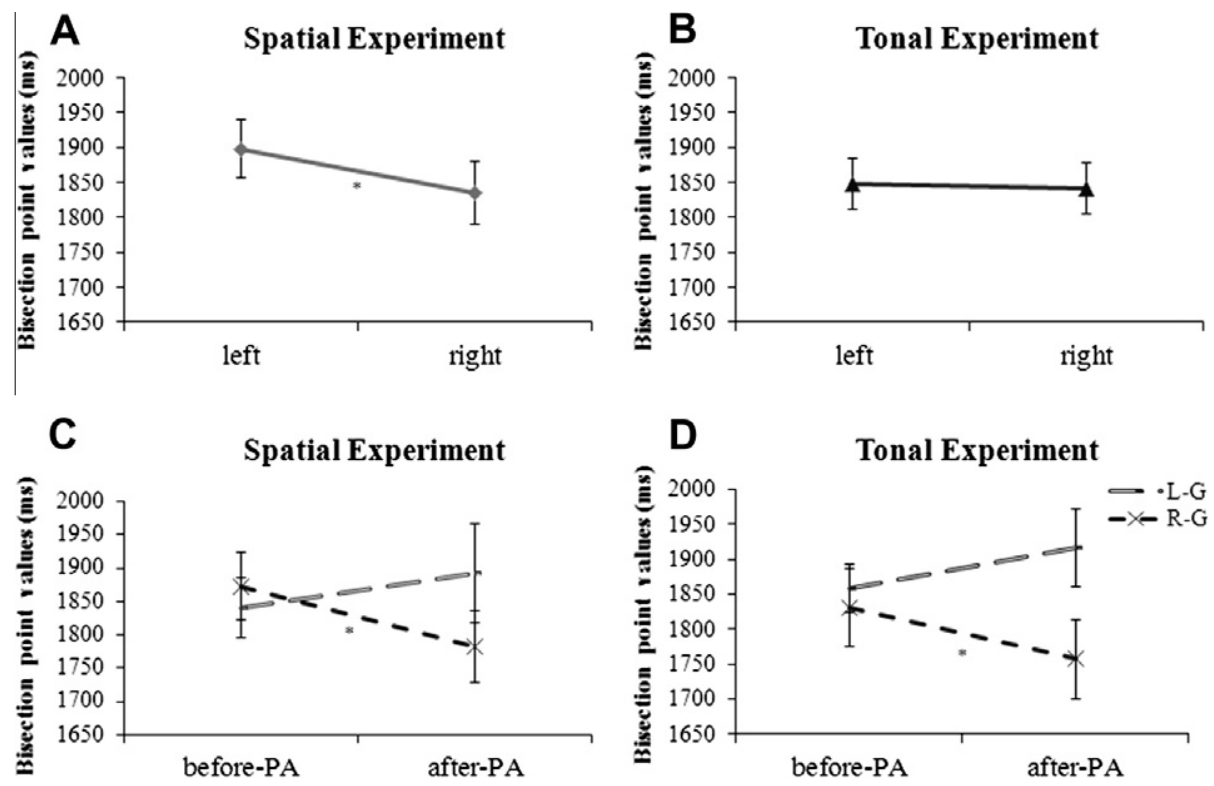

Fig. 1. Bisection point values in milliseconds (ms). Effect of Stimulus Position (left, right) in the Spatial experiment (A) and in the Tonal experiment (B). Effect of interaction between Group (L-G - leftward after-effect, R-G - rightward after-effect) and Condition (before-PA, after-PA) in the Spatial experiment (C) and in the Tonal experiment (D). Error bars indicate standard error of means. Asterisks indicate significant differences.

after-PA), Stimulus Position (left or right) and Stimulus Pitch (high tone or low tone) as within-subjects variables.

In the Spatial experiment, a significant main effect of Stimulus Position was found $[F(1,26)=8.21 ; p=0.008$; $\left.\eta_{p}^{2}=.240\right]$ showing relative underestimation for stimuli presented to the left compared to stimuli presented to the right (mean $=1878 \mathrm{~ms}, \mathrm{SE}=41$; mean $=1816 \mathrm{~ms}$, $\mathrm{SE}=40$ ). The interaction between Group and Condition was also significant $\left[F(1,26)=7.93 ; p=0.009 ; \eta_{p}^{2}=.234\right]$. Post hoc analysis revealed that the two groups were not statistically different before PA ( $L G=1841, \quad \mathrm{SE}=45$; $R G=1893, S E=74 ; p=0.16$ ), whereas after PA opposite effects were observed as a function of prismatic shifts, with the two groups providing significantly different bisection points (LG: 1873, SE = 50; RG: 1783, SE=55; p=0.02). However, the effect of PA on time was asymmetric: RG overestimated time after PA with respect to before PA $(p=0.005)$, whereas this difference was not significant for LG ( $p=0.37$; see Fig. $1 C$ ) despite a numerical trend toward time underestimation. No other main effect or interaction reached significance. In particular, there was no interaction involving Stimulus Position, revealing that a similar difference in duration estimation as a function of sound location was maintained after PA.

In the Tonal experiment, a significant interaction between Group and Condition was also found $[F(1,26)=$ $\left.6.35 ; p=0.02 ; \eta_{p}^{2}=.196\right]$. Post hoc analysis revealed that the two groups were not statistically different before PA ( $L G=1858, S E=34 ; G R=1830, S E=56 ; p=0.45$ ) whereas after PA opposite effects emerged as a function of prismatic shifts, with the two groups providing significantly different bisection points (LG: 1917, SE = 56; RG: 1757, $S E=57$; $p=0.0002$ ). Again, the effect of PA on time was asymmetric: the rightward after-effect group overestimated time after PA with respect to before PA ( $p=0.05)$, whereas this difference was not significant for leftward after-effect group ( $p=0.12$; see Fig. 1D) despite a numerical trend toward time underestimation. The absence of any main effect or interaction involving the Stimulus Position variable shows that PA did not enforce any difference in duration estimation as a function of sound location both before PA and after PA.

\subsection{Analysis on the Percentage of 'long' responses}

To validate further the findings obtained using estimated bisection point as dependant variable, we re-analyzed the data also in terms of percentage of 'long' responses for each stimulus duration, as a function of stimulus location and PA. For each experiment (Spatial and Tonal) we conducted an ANOVA on the percentage of "long" responses, using Group (LG = leftward after-effect group or RG = rightward after-effect group) as betweensubjects variable and Condition (before-PA vs. after-PA), Stimulus Position (left vs. right) and Intervals (1400, $1700,2000,2300,2600, \mathrm{~ms})$ as within-subjects variables.

Overall, the results were highly consistent with those reported in the previous analysis on bisection point (see Fig. 2). In the Spatial experiment, the effect of Stimulus Position was significant $\left[F(1,26)=5.69 ; p=0.03 ; \eta_{p}^{2}=.179\right]$. Overall, percentage of "long" responses was higher (overestimation of time) for right $(61 \%, \mathrm{SE}=8 \%)$ than left stimuli (58\%, SE = 8\%; Fig. 2A). The interaction between Stimulus Position and Intervals was also significant $[F(4,104)=$ $\left.2.73 ; p=0.03 ; \eta_{p}^{2}=.094\right]$. Post hoc analyses revealed that even if the percentage of "long" responses was higher for right stimuli than for left stimuli, this difference was significant for the middle interval (2000 ms) (right, 77\% vs left, 
A
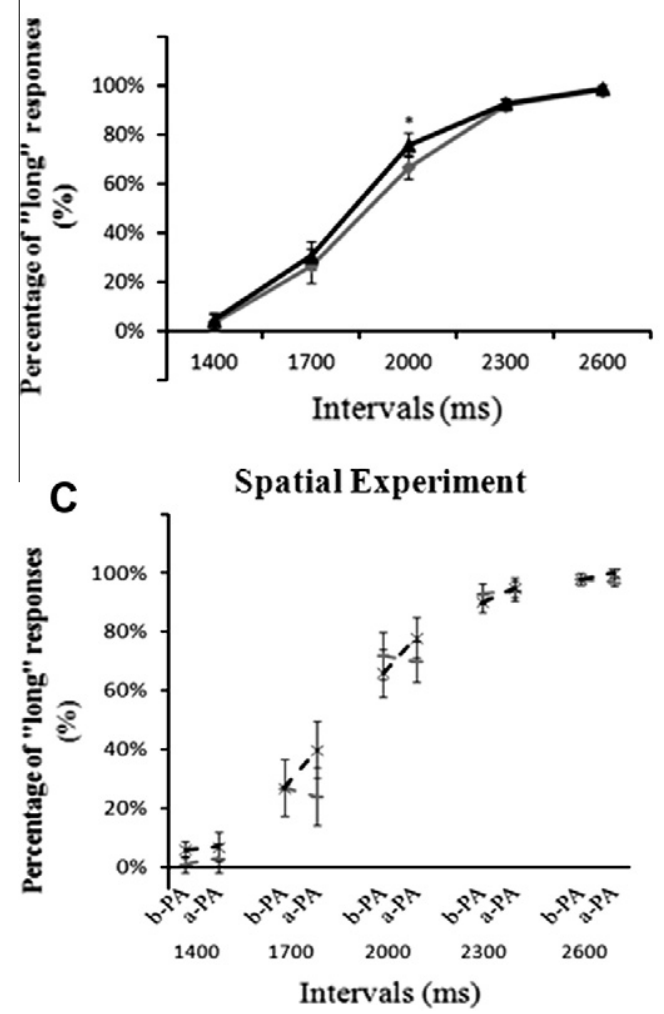

B

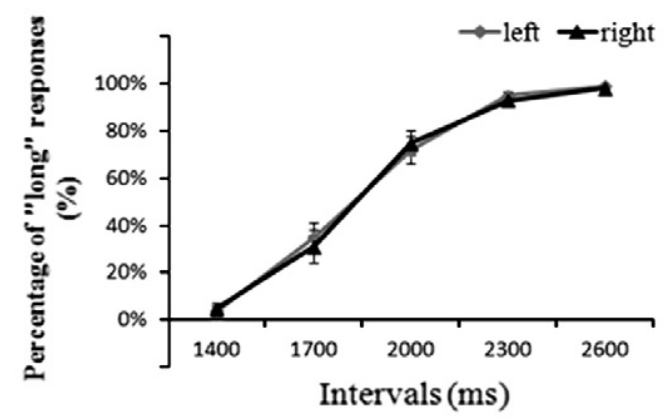

D

Tonal Experiment

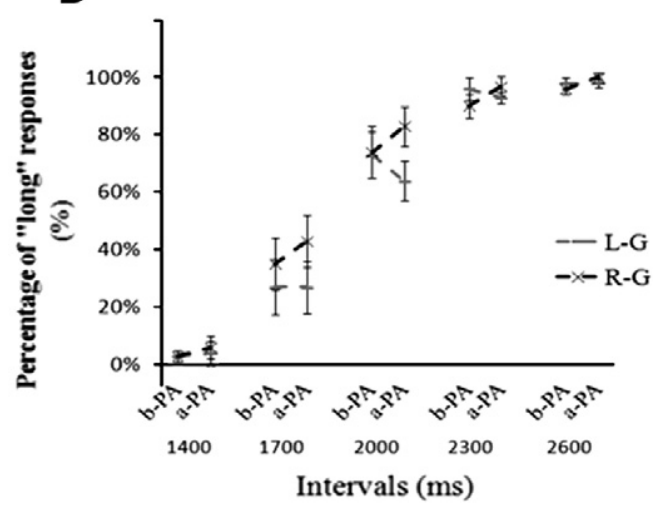

Fig. 2. Percentage of "long" responses (\%). Effect of the interaction between Stimulus Position (left, right) and Intervals (1400, 1700, 2000, 2300, 2600, ms) in the Spatial Experiment (A) and in the Tonal Experiment (B). Effect of the interaction between Group (L-G leftward after-effect, R-G-rightward aftereffect), Condition (b-PA = before-PA, a-PA = after-PA) and Intervals (1400, 1700, 2000, 2300, 2600, ms) in the Spatial Experiment (C) and in the Tonal Experiment (D). Error bars indicate standard error of means. Asterisks indicate significant differences.

$67 \%, \mathrm{p}<0.001)$, but not for the other intervals $(1400 \mathrm{~ms}$, $1700 \mathrm{~ms}, 2300 \mathrm{~ms}, 2600 \mathrm{~ms}, \mathrm{p}>0.07$ for all comparisons).

When we considered the effect of PA (Fig. 2C), we found a significant main effect of Condition $[F(1,26)=6.35$; $\left.p=0.02 ; \eta_{p}^{2}=.196\right]$, an interaction between Group and Condition $\left[F(1,26)=9.74 ; p=0.004 ; \eta_{p}^{2}=.273\right]$ and an interaction between Group, Condition and Intervals $\left[F(4,104)=2.97 ; p=0.02 ; \eta_{p}^{2}=.102\right]$. The percentage of "long" responses after PA (compared to before PA) was higher in the RG group than the LG group. This difference reached significance for the two central intervals ( $1700 \mathrm{~ms}, 41 \%$ vs $27 \%$; $2000 \mathrm{~ms}, 78 \%$ vs $66 \%$; $<<0.001$ for both comparisons). At the $1700 \mathrm{~ms}$ interval, the percentage of "long" responses after PA was also significantly higher for the RG group compared to the LG group (41\% vs $24 \%$, $\mathrm{p}=0.03$ ). No difference between before and after PA conditions emerged for the LG group, despite a trend toward lower percentage of "long" responses after PA relative to before PA at the two central intervals $(1700 \mathrm{~ms}$ and $2000 \mathrm{~ms}$ ). This confirms the asymmetry of the PA effect between groups documented in the bisection point analyses reported above.

In the Tonal experiment, the effect of Stimulus Position $(\mathrm{p}=0.53)$ and its interaction with other variables were not significant $(p>0.06)$ (Fig. $2 B)$. When we examined the effect of PA (Fig. 2D), we found a significant interaction between Group and Condition $[F(1,26)=6.46 ; p=0.02$; $\left.\eta_{p}^{2}=.199\right]$. Post hoc analysis revealed once again that the effect of PA on time estimation was asymmetric: the RG group showed a higher percentage of "long" responses (overestimation of time) after PA with respect to before $\mathrm{PA}(66 \%, \mathrm{SE}=11 \%$, vs $60 \%, \mathrm{p}=0.005)$, whereas this difference was not significant for LG group ( $57 \%$, SE $=11 \%$, vs $59 \%, \mathrm{p}=0.36)$. The interaction between Group and Intervals also reached significance $[F(4,104)=2.62 ; p=0.04$; $\left.\eta_{p}^{2}=.092\right]$, caused by higher percentage of "long" responses in RG relative to LG group, for the central intervals ( $1700 \mathrm{~ms}, 39 \%$ vs $27 \%, \mathrm{p}=0.03 ; 2000 \mathrm{~ms}, 79 \%$ vs $68 \%$, $\mathrm{p}=0.05$ ). No other main effect or interaction reached significance.

In sum, these results show that the spatial location of stimuli influences the percentage of "long" responses toward an overestimation of right stimuli relative to left stimuli, in the spatial but not in the tonal experiment. This effect of spatial location is significant for the very central interval of the distribution (2000 ms), suggesting that the spatial location exerts its effect in the point of maximum uncertainty when judging a time interval.

Furthermore, parallel to what found with the analysis on bisection point, prismatic adaptation influences the percentage of "long" responses in both the spatial and tonal experiment. This influence was asymmetric: prisms inducing 
a rightward after-effect significantly increased the percentage of "long" responses while prisms inducing a leftward after-effect did not significantly change the percentage of "long" responses despite a trend of means toward a decrease. Similarly, the effect of PA on time intervals was significantly evident at the central stimuli of the distribution, suggesting that the effects of a spatial manipulation (whatever spatial location or PA condition) occurs when the participant is mostly uncertain about the response when judging a time interval.

\subsection{Prismatic Adaptation}

To ensure that pre-PA/post-PA differences in time bisection task were due to the PA procedure we assessed the presence of both error reduction and after-effect.

To verify that participants showed error reduction as they adapted to the prisms, we conducted an ANOVA on the mean displacement (expressed as degrees of visual angle) of participants' visible pointing, with Group (LG vs RG) as a between-subjects variable and Condition (pre-exposure condition, first three trials of the exposure condition, last three trials of the exposure condition) as a within-subjects variable (more details on this procedure can be found in Frassinetti et al., 2002). Post hoc comparisons were conducted using the LSD test. The effect of Group was significant $\left[F(1,26)=80.41 ; p<0.0001 ; \eta_{p}^{2}=.756\right]$ : LG showed an overall pointing displacement to the right while RG showed an overall pointing displacement to the left $\left(0.376^{\circ}, \mathrm{SE}=0.17 ;-0.553^{\circ}, \mathrm{SE}=0.26\right)$, compatible with the rightward and leftward prismatic deviation respectively. The interaction between Group and Condition was significant $\left[F(2,52)=80.41 ; p<0.0001 ; \eta_{p}^{2}=.756\right]$ : post hoc analysis revealed that pointing displacement in pre-exposure condition and in first three trials of the exposure condition was significantly different both for LG $\left(0.000^{\circ}\right.$, $\left.\mathrm{SE}=0 ; 1.127^{\circ}, \mathrm{SE}=0.15 ; \mathrm{p}<0.0001\right)$ and for $\mathrm{RG}\left(0.000^{\circ}\right.$, $\left.\mathrm{SE}=0 ;-1.661^{\circ}, \mathrm{SE}=0.27 ; \mathrm{p}<0.0001\right)$, whereas it was not different from the pointing displacement in last three trials of the exposure condition ( $L G=0.000^{\circ}, \mathrm{p}=1 ; \mathrm{RG}=-0.000^{\circ}$, $\mathrm{p}=1$ ) (see Fig. 3A).

To verify the presence of an after-effect, we compared participants' displacement during invisible pointing in the pre-exposure and post-exposure conditions. An ANOVA on the mean displacement of invisible pointing responses was carried out with Group (LG vs RG) as a between-subjects variable and Condition (pre-exposure vs post-exposure) as a within-subjects variable. The interaction between Group and Condition was significant $[F(1,26)=$ 206.37, $p<0.0001 ; \eta_{p}^{2}=.888$ ] since as expected, LG and RG showed significant leftward and rightward deviations, respectively, in the post-exposure condition relative to the pre-exposure condition ( $\mathrm{LG}=-4.558^{\circ}, \mathrm{SE}=0.36$; vs $1.773^{\circ}, \mathrm{SE}=0.31, \mathrm{p}<0.0001 ; \mathrm{RG}=3.060^{\circ}, \mathrm{SE}=0.50 ; \mathrm{vS}-$ $0.867^{\circ}, \mathrm{SE}=0.42 ; \mathrm{p}<0.0001$ ) (see Fig. $3 \mathrm{~B}$ ).

\subsection{Correlational Analysis}

To assess possible relationships the effects on time perception documented above and the magnitude of PA (in terms of error reduction and after-effect), we conducted Pearson correlation analyses. As measure of PA effect on time we took the difference between bisection point before and after PA, separately for the Spatial and the Tonal experiment. Negative values indicate underestimation after PA compared to before PA, whereas positive values indicate overestimation. As measure of error reduction during PA, we computed the difference between the mean pointing displacement in the first three trials minus the mean pointing displacement in the last three trials in the

\section{A - Error reduction}

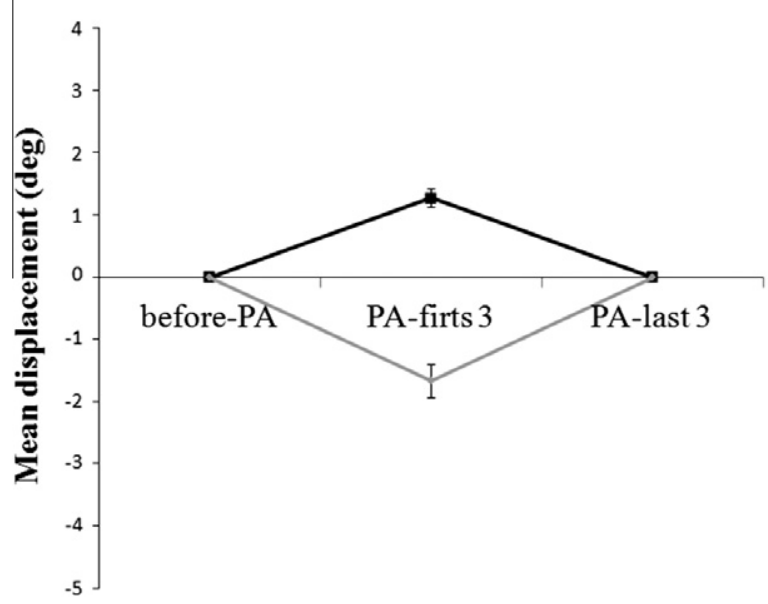

B - After-effect

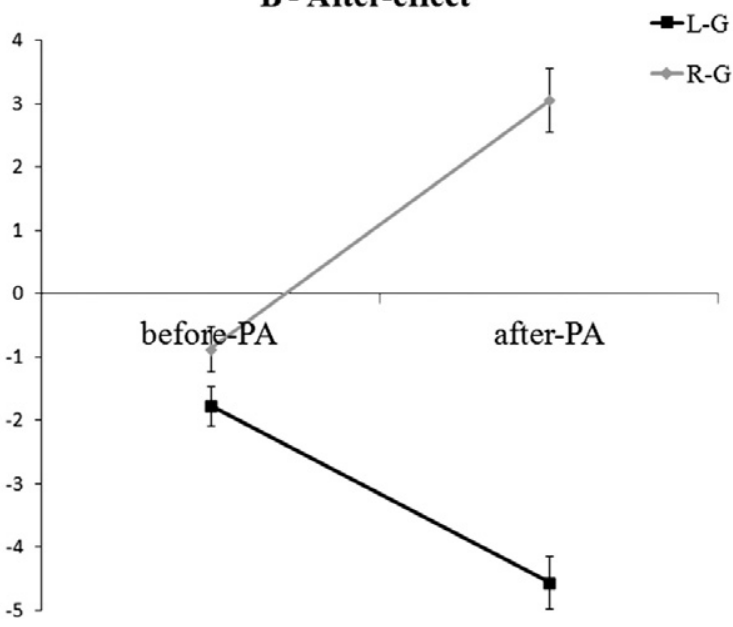

Fig. 3. Mean displacement in degrees of visual angle (deg) of pointing responses in participants with a leftward after-effect (LG) and in participants with a rightward after-effect (RG) induced by rightward and leftward prism adaptation, respectively. Results demonstrating Error reduction shown in (A), which represents mean displacement of participants' visible pointing before prismatic adaptation (before-PA) and in the first three (PA-first 3 ) and last three trials (PA-last 3) during PA. Results demonstrating After-effect of PA shown in (B), which represents mean displacement of participants' invisible pointing before (before-PA) and after PA (after-PA). Negative values indicate leftward pointing displacement with respect to the target's actual location; positive values indicate rightward pointing displacement. Error bars indicate standard error of means. 
exposure condition. Since pointing displacement in the last three trials was always zero, this difference corresponds to the mean pointing displacement in the first three trials (i.e., the starting pointing displacement). As measure of after-effect, we took pointing displacement in the postexposure condition.

The analysis revealed a negative correlation between starting pointing displacement and after-effect $(\mathrm{r}=-0.82$; $\mathrm{p}<0.0001$ ) suggesting that the larger the pointing displacement in the direction of lenses deviation, the larger the after-effect in the opposite direction. Most interestingly, the analysis indicated a negative correlation between starting pointing displacement and auditory duration processing both in the Spatial $(\mathrm{r}=-0.60 ; \mathrm{p}=$ $0.001)$ and in the Tonal $(r=-0.43 ; p=0.02)$ experiment (see Fig. 4A). Specifically, the larger the pointing displacement in the direction of lenses deviation, the larger the effect of PA on time. When pointing displacement was positive (lenses to the right inducing leftward after-effect) the effect on time was an underestimation, when pointing displacement was negative (lenses to the left inducing rightward after-effect) the effect on time was an overestimation. Finally, a positive correlation was found between after-effect and time both in Spatial $(r=0.52 ; \mathrm{p}=0.005)$ and Tonal $(\mathrm{r}=0.38 ; \mathrm{p}=0.05)$ experiment (see Fig. 4B): the larger the after-effect to the left the larger the underestimation, the larger the after-effect to the right the larger the overestimation.

\section{Discussion}

The present study examined the spatial representation of auditory time focusing on two novel aspects: first, the role of spatial vs. tonal coding of sound in enhancing such a representation; second, the role of prismatic adaptation as modulatory effect. If auditory time is spatially represented on a mental time line (MTL) left-to-right oriented, underestimation of stimuli presented to the left and overestimation of stimuli presented to the right was expected. The present findings confirmed this prediction by showing that duration of auditory stimuli was underestimated when they were presented to the left side of the participant, with respect to when they occurred on the right side. Most important, this effect was evident only when the auditory stimuli required previous spatial encoding (Spatial experiment), but not when they required tonal encoding (Tonal experiment). When participants were asked to respond taking into account the stimulus spatial
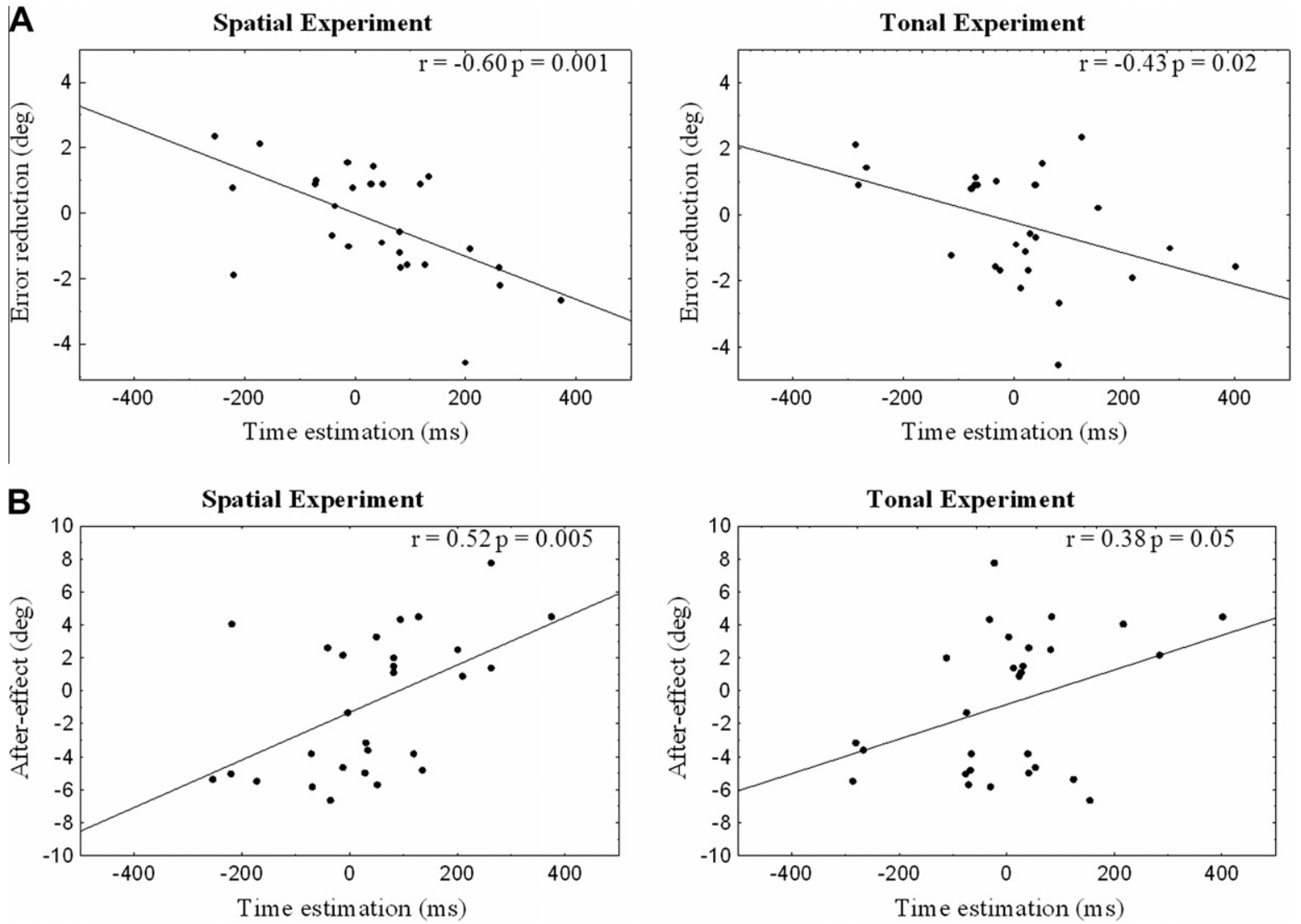

Fig. 4. Correlational analysis between: (A) Error reduction (in deg) and the effect of PA on time estimation expressed in millisecond (ms), in the Spatial experiment and in the Tonal experiment; (B) After-effect (deg) and the effect of PA on time estimation (ms), in the Spatial experiment and in the Tonal experiment. Each graph reports $r$ and $p$ values. Note that the effect of PA on time estimation was calculated for each experiment as the difference in bisection point before and after PA. Negative values mean underestimation of time; positive values mean overestimation of time. 
location (left or right), space influenced duration estimation. This suggests that when auditory stimuli were coded as 'left' or 'right', this information about their location in external space was reflected in their representation on the mental temporal line. As a result, duration of left stimuli was underestimated with respect to the duration of right ones. By contrast, when subjects were asked to respond taking into account the stimulus tonal frequency, the actual location in space (left or right) did not influence duration estimation.

\subsection{Spatial coding of auditory time is task-dependent, but independent of motor response}

These findings corroborate and extend the results of a recent work on auditory time by Ishihara et al. (2008), showing that left-side responses were faster for early-onset timing than late-onset timing, whereas right-side responses were faster for late-onset timing than early-onset timing. These results supported the idea that time and space metrics interact in action (Walsh, 2003; Bueti \& Walsh, 2009). The present work extends the findings of Ishihara et al. (2008) in several ways. First, we show that spatial interference on time processing for sounds requires previous spatial encoding of the stimulus. The auditory system is not inherently spatial, because information is initially encoded tonotopically and space is not immediately available on receptor surface (Barker et al., 2011; Hall \& Plack, 2009). To encode the spatial location of auditory stimuli the brain implements additional processes, based on interaural and monaural auditory cues (Blauert \& Lindemann, 1986). As a result, the output of this computation is typically more complex and less precise than the localization of a visual stimulus. Our results indicate that the spatial representation of auditory time emerges more strongly whenever a spatial encoding of the auditory stimuli is enforced. Second, differently from Ishihara et al.'s study, we examined duration instead of timing expectancy. Third, we did not use a motor response and our findings show that a spatial representation of auditory time emerges also without interactions with a spatial motor response. The observation that time and space can interact even in the absence of motor actions is particularly relevant. One possible explanation is that the brain develops spatial metric maps during action interactions with the environment. Once this metric system has completed its development, it is used as a code to compute other cognitive operations and not just for measuring quantity dimensions useful for action (Bueti \& Walsh, 2009). The existence of tight links between motor spatial maps and cognitive spatial maps has been widely demonstrated. On one hand, the findings described so far, including the present study, indicate that a manipulation of spatial attention influences the spatial representation of time both with or without motor response (Vicario et al., 2007; Vicario et al., 2008; Oliveri et al., 2009b). On the other hand, the reversed condition has also been demonstrated: a manipulation of the representation of time influences spatial attention orienting and motor preparation in space. For example, Ouellet et al. (2010) examined the nature of the space-time conceptual metaphor, by testing whether the temporal meaning of words presented centrally on screen can orient spatial attention and/or prime a congruent left/right motor response. They found that the mere exposure to past or future words both oriented attention and primed motor responses to left or right space, respectively. Similarly, a recent ERP study by Vallesi, McIntosh, and Stuss (2011) found that centrally presented time intervals, pre-activated the corresponding motor cortex and speeded up a response mapping compatible with a short/left and long/ right order. This evidence of a multidirectional influence among spatial attention orienting, spatial representation of time and spatial motor responses, suggests that spatial metric is a very centralized representation that is employed for spatial operations at other different levels of the cognitive system, and that a perturbation in the spatial metric at one level reflects in the same perturbation at all other levels.

\subsection{Prismatic adaptation modulates spatial coding of auditory time}

A further novel result of the present study is that representation of auditory durations was modulated by prismatic adaptation. More precisely, leftward and rightward attentional shift induced by PA resulted in opposite effects on the estimation of auditory time intervals, regardless of the side of space in which stimuli were presented (left or right) and irrespective of whether the task required a spatial or a tonal coding of the sound. Strikingly, our correlational analysis indicates a relationship between PA parameters (namely, starting pointing displacement and after-effect) and the PA effect on duration processing, both in the Spatial and in the Tonal experiment. Finally, we found that this effect was asymmetric in magnitude. PA affected time estimation more strongly when attention was shifted to the right, compared to when it was shifted to the left.

The strong effects of PA on spatial coding of auditory time has several relevant implications. First, it provides evidence that adaptation of visuo-motor coordination can affect performance on a sensory modality (audition) that is not directly implicated in PA. In this respect, there is a parallel between PA effects on auditory time and PA effects on auditory spatial processing (Eramudugolla et al., 2010; Jacquin-Courtois et al., 2010). In brain-damaged patients with visual and auditory neglect, Eramudugolla et al. (2010) found that the overall auditory detection performance improved after PA relative to before PA. Similarly, Jacquin-Courtois et al. (2010) found that PA improved discrimination of auditory stimuli delivered at the contralesional ear. Jacquin-Courtois and colleagues explain their data suggesting that the lateralized remapping of visuo-motor information induced by prism could subsequently alter attention orienting in the auditory modality. In our opinion, and in accord with the present data showing an effect of prismatic adaptation on auditory time stimuli, the results of Jacquin-Courtois et al., suggest that PA transferred benefit to the auditory modality that is orthogonal to the visual, proprioceptive and motor modalities directly implicated in the visuo-motor adaptive process. This implies that effects of PA can extend to 
unexposed sensory systems, suggesting implication of a supra-modal effect. Once the sensory representation of duration is translated at high cognitive level in a spatial representation, it is not auditory featured anymore. In this sense, PA may have not affected audition but rather a spatial supra-modal representation of temporal stimuli. In this respect, it is important to note that a shift of spatial attention to opposite sides have produced opposite effects on time, independently on the stimuli location (left or right) and independently on the kind of encoding (spatial or tonal). This result reinforces the hypothesis that PA affects the spatial representation of any auditory duration once it has been encoded and translated into a cognitive one. The resulting effect of PA is similar to a "distortion" of the representation of any auditory duration, by shortening or extending it according with the leftward or rightward attentional deviation.

Concerning the asymmetric effects of PA deviation on auditory time, it should be emphasized that such asymmetries are not new in the literature on PA. Previous studies showed that rightward shifts of spatial attention induced by prismatic adaptation are stronger than the leftward ones, as we also document here (Goedert, Leblanc, Tsai, \& Barrett, 2010; Colent, Pisella, Bernieri, Rode, \& Rossetti, 2000). Colent et al. (2000) demonstrated that after a session of PA shifting spatial attention to the right, participants bisected horizontal lines more to the right relative to before PA. The opposite effect was not found with leftward PA deviation. Since rightward line bisection is a typical behavior of patients with hemispatial neglect, the authors considered their results in terms of a simulation of neglect in neurologically healthy individuals. Because neglect syndrome is more likely to occur after right rather than left hemispherical lesions, inducing a rightward bias of spatial attention, Colent and colleagues proposed that the asymmetrical effect of rightward and leftward PA on space might reflect an inherent bias of the brain's structural organization in directing attention to the right. Accordingly, some studies suggest that neglect (post-lesion in patients) and pseudo-neglect (physiological bias that is observed in some tasks in healthy subjects) could be expressions of common cognitive and neural mechanisms (McCourt \& Jewell, 1999). In other words, cognitive performances of healthy subjects after PA could be considered as correct approximation of a neglect-like behavior, with common main characteristics (i.e. directional bias, directional specificity, predominance of perceptive effects; Michel et al., 2003).

An explanation that is not in contrast but complementary to the last one, is that the left-to-right shift of spatial attention (and of temporal representation) is easier to be induced than the right-to-left shift, because it has been acquired more extensively in a culture adopting a left-toright writing/reading system. Therefore it would be difficult to overcome with a short prismatic adaptation training phase. Our participants were all Italian native language speakers, learning exclusively a left-to-right writing/reading habit. Supporting this explanation there is evidence about the strong influence of the writing/reading habits on the congruency effects involving space (Dehaene, Bossini, \& Giraux, 1993). Alternatively, the asymmetrical results on the line bisection task could be attributed to sensory-motor after-effect. However, this can be excluded because in the present study, similarly to the study of Colent et al. (2000), we observed symmetrical sensory-motor after-effects, regardless of prismatic deviation side, despite an asymmetrical effect on the representation of duration.

Whatever the interpretation of the asymmetry of PA effects found in the present study, our findings strongly suggest that the engaged process concerns some supramodal level of spatial representations. These effects confirm that sensory-motor integration can structure spatial cognition and hence that sensori-motor and cognitive representations of space are not fully dissociated, supporting our hypothesis that PA affected the high cognitive spatial representation of time for auditory modality.

In conclusion this study indicates that the spatial representation of auditory time emerges more strongly whenever a spatial encoding of the auditory stimuli is enforced and that this spatial representation of auditory time can be modulated by a shift of spatial attention obtained through the PA procedure.

\section{Acknowledgements}

This work was supported by grants from RFO (Ministry of University and Research) to Francesca Frassinetti. Francesco Pavani was supported by grants from the Italian Ministry of University and Research (PRIN), and from the Fondazione Cassa di Risparmio di Trento e Rovereto.

Thanks to Stella Torrenti for her contribution in collecting data.

\section{References}

Allan, L. G. (2002). The location and interpretation of the bisection point. Quarterly Journal of Experimental Psychology, 55B, 43-60.

Angeli, V., Benassi, M. G., \& Làdavas, E. (2004). Recovery of oculo-motor bias in neglect patients after prism adaptation. Neuropsychologia, 42(9), 1223-1234.

Barker, D., Plack, C. J., \& Hall, D. A. (2011). Human auditory cortical responses to pitch and to pitch strength. NeuroReport, 22(3), 111-115.

Blauert, J., \& Lindemann, W. (1986). Spatial mapping of intracrania auditory events for various degrees of interaural coherence. Journal of the Acoustical Society of America, 79(3), 806-813.

Bueti, D., \& Walsh, V. (2009). The parietal cortex and the representation of time, space, number and other magnitudes. Philosophical Transactions of the Royal Society B: Biological Sciences, 364(1525), 1831-1840.

Calabria, M., Jacquin-Courtois, S., Miozzo, A., Rossetti, Y., Padovani, A., Cotelli, M., et al. (2011). Time perception in spatial neglect: a distorted representation? Neuropsychology, 25(2), 193-200.

Colent, C., Pisella, L., Bernieri, C., Rode, G., \& Rossetti, Y. (2000). Cognitive bias induced by visuo-motor adaptation to prisms: a simulation of unilateral neglect in normal individuals? NeuroReport, 11(9), 1899-1902.

Dehaene, S., Bossini, S., \& Giraux, P. (1993). The mental representation of parity and number magnitude. Journal of Experimental Psychology: General, 122(3), 371-396.

Deouell, L. Y., \& Soroker, N. (2000). What is extinguished in auditory extinction? NeuroReport, 11(13), 3059-3062.

Eramudugolla, R., Boyce, A., Irvine, D. R., \& Mattingley, J. B. (2010). Effects of prismatic adaptation on spatial gradients in unilateral neglect: A comparison of visual and auditory target detection with central attentional load. Neuropsychologia, 48(9), 2681-2692.

Frassinetti, F., Angeli, V., Meneghello, F., Avanzi, S., \& Làdavas, E. (2002). Long-lasting amelioration of visuospatial neglect by prism adaptation. Brain, 125(Pt 3), 608-623.

Frassinetti, F., Magnani, B., \& Oliveri, M. (2009). Prismatic lenses shift time perception. Psychological Science, 20(8), 949-954. 
Gallistel, C. R., \& Gibbon, J. (2000). Time, rate, and conditioning. Psychological Review, 107(2), 89-344.

Goedert, K. M., Leblanc, A., Tsai, S. W., \& Barrett, A. M. (2010). Asymmetrical effects of adaptation to left- and right-shifting prisms depends on pre-existing attentional biases. Journal of the International Neuropsychological Society, 16(5), 795-804.

Hall, D. A., \& Plack, C. J. (2009). Pitch processing sites in the human auditory brain. Cerebral Cortex, 19(3), 576-585

Ishihara, M., Keller, P. E., Rossetti, Y., \& Prinz, W. (2008). Horizontal spatial representations of time: Evidence for the STEARC effect. Cortex, 44(4), 454-461.

Jacquin-Courtois, S., Rode, G., Pavani, F., O'Shea, J., Giard, M. H., Boisson, D., et al. (2010). Effect of prism adaptation on left dichotic listening deficit in neglect patients: glasses to hear better? Brain, 133(Pt 3), 895-908.

Kopec, C. D., \& Brody, C. D. (2010). Human performance on the temporal bisection task. Brain and Cognition, 74(3), 62-72.

Lee, C. C., \& Middlebrooks, J. C. (2011). Auditory cortex spatial sensitivity sharpens during task performance. Nature Neuroscience, 14(1), $108-114$.

Lewis, P. A., \& Miall, R. C. (2003). Distinct systems for automatic and cognitively controlled time measurement: evidence from neuroimaging. Current Opinion in Neurobiology, 13(2), 250-255.

McCourt, M. E., \& Jewell, G. (1999). Visuospatial attention in line bisection: stimulus modulation of pseudoneglect. Neuropsychologia, 37(7), 843-855.

Meck, W. H. (2005). Neuropsychology of timing and time perception. Brain and Cognition, 58(1), 1-8.

Michel, C., Pisella, L., Halligan, P. W., Luauté, J., Rode, G., Boisson, D., et al. (2003). Simulating unilateral neglect in normals using prism adaptation: implications for theory. Neuropsychologia, 41(1), 25-39.

Oliveri, M., Koch, G., \& Caltagirone, C. (2009a). Spatial-temporal interactions in the human brain. Experimental Brain Research, 195(4), 489-497.

Oliveri, M., Koch, G., Salerno, S., Torriero, S., Lo Gerfo, E., \& Caltagirone, C. (2009b). Representation of time intervals in the right posterior parietal cortex: implications for a mental time line. Neuroimage, 46(4), 1173-1179.
Ouellet, M., Santiago, J., Funes, M. J., \& Lupiáñez, J. (2010). Thinking about the future moves attention to the right. Journal of Experimental Psychology: Human Perception and Performance, 36(1), 17-24.

Pavani, F., Làdavas, E., \& Driver, J. (2002). Selective deficit of auditory localisation in patients with visuospatial neglect. Neuropsychologia, 40(3), 291-301.

Populin, L. C., \& Rajala, A. Z. (2010). Time course of allocation of spatial attention by acoustic cues in non-human primates. European Journal of Neuroscience, 32(6), 1040-1048.

Rode, G., Rossetti, Y., Li, L., \& Boisson, D. (1998). Improvement of mental imagery after prism exposure in neglect: a case study. Behavioral Neurology, 11(4), 251-258.

Rossetti, Y., Jacquin-Courtois, S., Rode, G., Ota, H., Michel, C., \& Boisson, D. (2004). Does action make the link between number and space representation? Visuo-manual adaptation improves number bisection in unilateral neglect. Psychological Science, 15(6), 426-430.

Serino, A., Angeli, V., Frassinetti, F., \& Làdavas, E. (2006). Mechanisms underlying neglect recovery after prism adaptation. Neuropsychologia, 44, 1068-1078.

Vallesi, A., Binns, M. A., \& Shallice, T. (2008). An effect of spatial-temporal association of response codes: understanding the cognitive representations of time. Cognition, 107(2), 501-527.

Vallesi, A., McIntosh, A. R., \& Stuss, D. T. (2011). How time modulates spatial responses. Cortex, 47(2), 148-156.

Vicario, C. M., Caltagirone, C., \& Oliveri, M. (2007). Optokinetic stimulation affects temporal estimation in healthy humans. Brain and Cognition, 64(1), 68-73.

Vicario, C. M., Pecoraro, P., Turriziani, P., Koch, G., Caltagirone, C., \& Oliveri, M. (2008). Relativistic compression and expansion of experiential time in the left and right space. PLoS One, 3(3), e1716.

Walsh, V. (2003). A theory of magnitude: common cortical metrics of time, space and quantity. Trends in Cognitive Science, 7(11), 483-488.

Wearden, J. H. (1991). Human performance on an analogue of an interval bisection task. Quarterly Journal of Experimental Psychology B, 43(1), 59-81.

Wearden, J. H., \& Ferrara, A. (1995). Stimulus spacing effects in temporal bisection by humans. Quarterly Journal of Experimental Psychology B, 48(4), 289-310. 